\title{
A new replication medium enables a rapid identification of $\varphi x-174$ virus by synchronous fluorescence of Tryptophan.
}

Yair Farber ${ }^{1,2 a^{*}}$, Yaniv Shlosberg ${ }^{3,4 a}$, Noam Adir ${ }^{3,4}$, Israel Schechter ${ }^{4}$, and Robert Armon ${ }^{5}$.

\author{
*Corresponding author: yair.farebr@mx.kinneret.ac.il \\ ${ }^{a}$ Contributed equally \\ ${ }^{1}$ Quality and Reliability Engineering Department, Kinneret Academic College, Zemach 1513200, Israel \\ ${ }^{2}$ Grand Water Research Institute, Technion, Haifa 3200003, Israel \\ ${ }^{3}$ Grand Technion Energy Program, Technion, Haifa 32000, Israel \\ ${ }^{4}$ Schulich Faculty of Chemistry, Technion, Haifa 32000, Israel \\ ${ }^{5}$ Faculty of Civil and Environmental Engineering, Technion, Haifa 32000, Israel
}

\begin{abstract}
Development of rapid methods for identification of bacteriophages based on their intrinsic fluorescence is challenging. Pure bacteriophages may be detected based on the strong fluorescence of the amino acid Tryptophan that exist in their proteins. Nevertheless, Tryptophan is a molecule that also exist in high quantities in the bacterial hosts and their cultivation media. In this work, we show that simple separation of the bacteriophage $\varphi x-174$ from its E.coli host (grown on standard cultivation medium) by filtration is not sufficient for its identification based on the intrinsic fluorescence of its Tryptophan content. This is mostly because of the tryptophan residues that derive from the cultivation medium. We fabricate a new cultivation medium that does not have any significant fluorescence overlap with Tryptophan. By utilization of this new cultivation medium, we can identify $\varphi x-174$ based on the spectral fingerprint of its intrinsic Tryptophan content by synchronous fluorescence measurements.
\end{abstract}

\section{Introduction}

Since the dawn of humanity, pathogens were always considered as a major risk for the human health. Over the years different efficient anti-bacterial agents were successfully developed such as anti-biotics and various peptide based drugs[1]. However, in the case of viral attack many 
infective diseases have remained cureless.[2] In the recent year, global pandemic (caused by SARS-CoV-2 virus) has spread all around the world causing millions of deaths and severe illnesses. [3] Rather than finding a cure, one of the main tools in the struggle against viral plagues is the detection of the viral presence in infected people. Such a diagnosis enables to keep them isolated and, in this way, to prevent them from infecting additional people. Therefore, the development of sensitive rapid viral identification method is highly important.

Classical identification methods utilize bacterial or mammalian cells as host for the viruses. The cell lysis that is caused by the viruses forms discrete visible zones (plaques) that can be detected by microscopic observation. [4] Purification of viruses from their host cell debris and other contaminants was previously done by different methods of density gradient centrifugation[5], ultrafiltration[6], and different chromatographic methods.[7, 8] The small sizes of viruses makes them invisible to simple microscopic observation and can be done by transmission electron microscopy (TEM) [9] or by utilization of fluorescent labelling agents under advanced microscopic instrumentation[10]. Determination of viral structures was extensively investigated by X-ray crystallography, NMR and cryo-TEM. [11, 12]. While a high purification degree of viruses is essential for their structural and fundamental studies, it may be time consuming or require the utilization of expensive instruments. Therefore, such methods may not be feasible when a rapid identification is required. Identification of virus can be done without an extensive purification process by utilization of biosensors[13-17]. In most cases such biosensors are based on immunoassays in which unique antibodies can target specific viruses. Identification of the bound antibodies can be done by simple gelelectrophoresis or by immunofluorescence methods. [18].

Another identification method which is very precise is the next generation sequencing (NGS) that can sequence the whole genome of the virus in a relatively short time. [19]A big advantage of sequencing the whole viral genome derives from the ability to identify unexpected mutations and being able to follow evolutionary evolvement. [20] Nevertheless, whole genome sequencing is still relatively expensive for a large-scale usage.

A cheaper method for detection that is extensively used in the recent years is polymerase chain reaction (PCR) [11, 12]. This method can detect viruses by amplification of specific sequences in the viral genome. An enhanced sensitivity was achieved by the developed of loop-mediated isothermal amplification (LAMP) and recombinase polymerase amplification (RPA) [21-25]. 
Different Spectroscopic methods were reported to identify isolated viruses. Raman spectroscopy was reported to be able to identify viruses [26] and also help determining their characterize their structure [27-29]. The amino acids Tryptophan, Tyrosine and Phenylalanine have an intrinsic fluorescence and can be used for the detection of isolated viruses. [30] Intrisic fluorescence can also be measured from nucleic acids, however is relatively low, and in many cases may not be sufficient to be detected [31]. Nevertheless, the fluorescence intensity of nucleic acids can be detected by utilization of labeling agents such as SYBR dyes, that significantly amplified it. [32]

Several scanning methods are commonly available in commercial fluorimeters. The scanning method which is most extensively used is the emission scan in which the excitation is being done with a single wavelength and a wide range of emission wavelengths is collected. A more advanced scanning method is synchronous fluorescence (SF). [33, 34] In this scanning method, both the excitation and emission wavelengths are scanned simultaneously while keeping a constant wavelength interval $(\Delta \lambda)$ between them. When the wavelength interval $\Delta \lambda$ between the excitation and emission wavelength is chosen properly, the resulting spectrum will show one or several features that are much more resolvable than those in the conventional fluorescence emission scan. The maximum fluorescence intensity for a particular component occurs when $\Delta \lambda$ corresponds to the difference between the wavelengths of the excitation and emission maxima for that component $[35,36]$. By narrowing the spectral bands, contraction of the spectral range, and simplification of the emission spectra - overlapping of spectra can be minimized $[37,38]$. While synchronous fluorescence methods have demonstrated as rapid tool for bacterial classification and identification [39-42], and even distinguishing between live and dead bacteria [43], to our best knowledge, no rapid identification of viruses by intrinsic synchronous fluorescence was reported.

Although fluorescence spectroscopy has a great potential for rapid identification of pure viruses based the intrinsic fluorescence of their amino acids, it is not compatible for matrixes with many biological compounds that are also consist of the same amino acids. In this work, we report a novel composition of a medium for the cultivation of the bacterial host of the virus $\varphi x$ 174, whose fluorescence contribution does not have any significant overlap with tryptophan. We show that this medium enables the identification of the spectral fingerprint of $\varphi x-174$ by a simple by a simple filtration followed by a synchronous fluorescence measurement.

\section{Results}




\section{Optimization of $\Delta \lambda$ for synchronous fluorescence measurements of Tryptophan}

One of the main native fluorescence contributors in biological components is the amino acid Tryptophan (Trp). We wished to assess the possibility to use the SF spectra of Trp as a fingerprint for identification of $\varphi x-174$. For this purpose, we first investigated optimal the measurement optimal $\Delta \lambda$ that will give the maximal intensity for Trp. Pure Trp solution $(1 \mu \mathrm{M})$ was measured using increasing $\Delta \lambda$ values. The highest intensity was obtained at $\Delta \lambda=60 \mathrm{~nm}$ (Fig. 1).

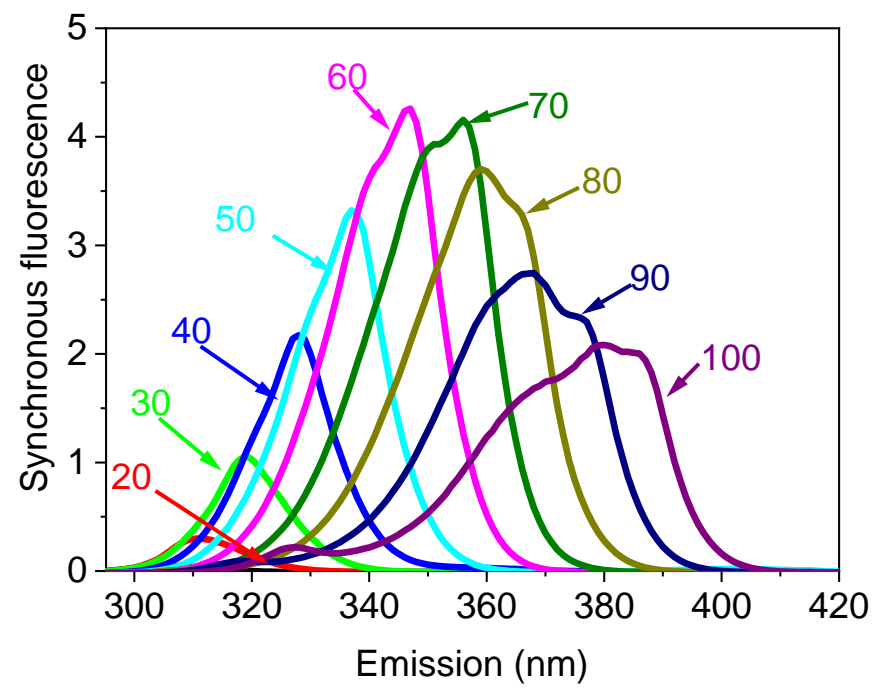

Fig. 1 - SF measurements of pure Trp. SF spectra of pure TRP was measured using increasing $\Delta \lambda$ values between 20 $100 \mathrm{~nm}$. The maximal intensity was obtained at $\Delta \lambda=60 \mathrm{~nm}$. $\Delta \lambda=20 \mathrm{~nm}$ (red), $30 \mathrm{~nm}$ (light green), $40 \mathrm{~nm}$ (blue), $50 \mathrm{~nm}$ (cyan), $60 \mathrm{~nm}$ (magenta), $70 \mathrm{~nm}$ (dark green), $80 \mathrm{~nm}$ (dark yellow), $90 \mathrm{~nm}$ (dark blue) and $100 \mathrm{~nm}$ (purple).

\section{Tryptophan residues in the bacterial media limits the detection of $\varphi x-174$}

A frequently used method for replication of $\varphi x-174$ in in petri dishes is called the double agar layer method. [44] In this method the virus is replicated on an E.coli layer that is grown on top of a gel bacterial substrate. One of the most frequently used substrates is Nutrient Agar (NA). Whole bacterial cells can be easily separated from $\varphi x-174$ by quick simple filtration or centrifugation. However, peptides ore proteins which consist of Trp may also originate form lysed bacterial cells or diffuse from the lower layer of the bacterial substrate. In this case, the separation from the virus is harder to perform. We wished to explore whether lysed cells and diffused molecules from the substrate limit the possibility to identify $\varphi x-174$ based on its Trp content. $\varphi x-174$ plaques were replicated on an E.coli layer on top of NA agar. The $\varphi x-174$ were extracted by ammonium acetate buffer for $10 \mathrm{~min}$. The supernatant was removed and filtrated through $0.2 \mu \mathrm{m}$ filter. The same procedure was repeated for petri dishes without $\varphi x-174$. SF of the filtrates were measured $\left(\Delta \lambda=60 \mathrm{~nm}, \lambda_{\mathrm{em}}(280-450 \mathrm{~nm})\right.$ (Figure. 3). Big peaks with maximal emission of 2.43 and 3.53 were obtained for the filtrates with and without $\varphi x-174$ respectively. Fitting analysis of the filtrate's peaks and pure Trp was calculated by 
multiple linear regression showing a good fit with RSS values of 0.11 and 0.17 with and without $\varphi x$ 174 respectively. (Figure. S1). The good fit indicates that the fluorescence in both spectra are the spectral fingerprint of Trp. The obtained results show that the high fluorescence contribution of the NA substrate or lysed bacterial cells that severely overlaps with the net fluorescence that originates from $\varphi x-17$. This overlap disable the ability for identification based on its Trp content.

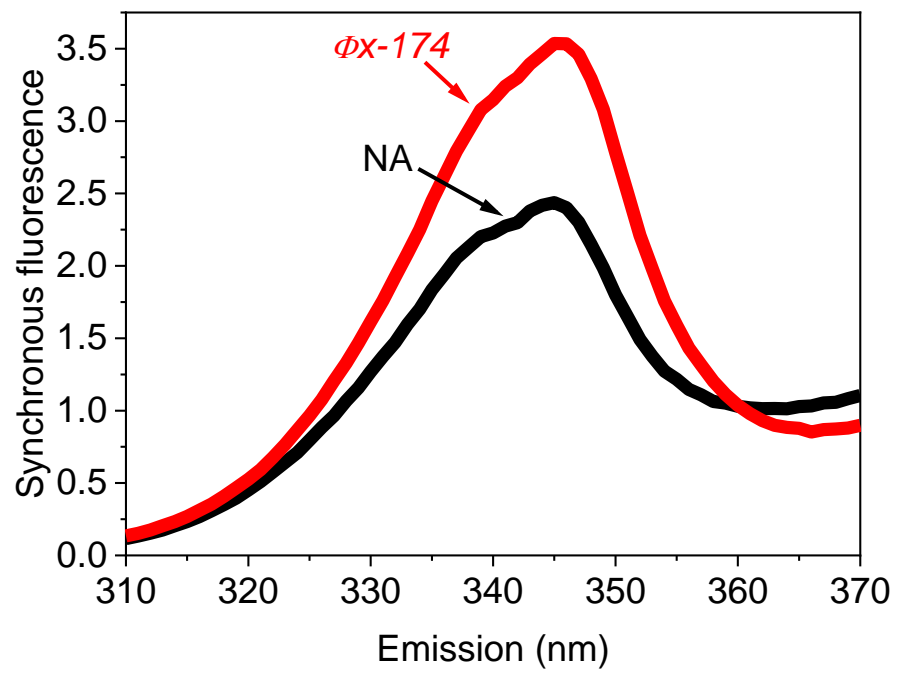

Fig. 2 - Tryptophan residues in the bacterial media limits the detection of $\varphi x-174$. SF spectra of E.coli extracts after filtration with and without $\varphi x-174\left(\Delta \lambda=60 \mathrm{~nm}, \lambda_{\mathrm{Em}}=310-370 \mathrm{~nm}\right)$. Although the intensity of $\varphi x-174$ was significantly higher, the spectral fingerprint of Trp was obtained in both spectra. SF of extracts filtrates without $\varphi x-174$ (black), and with $\varphi x-174$ (red).

\section{Preparation of a low Trp content medium}

In order to decrease the Trp fluorescence contribution that derives from the bacterial cultivation medium, we prepared a new medium which consist less Trp than in NA. The main ingredients of the low Trp substrate (LTS) were Agar, glucose, salts mixture and yeast extracts (for more details see experimental section). E.coli was successfully cultivated top of the LTS to form a uniformed layer.

Next, we wished to estimate the spectral interference of each individual group of ingredients and the mixture of all ingredients on spectral overlap with Trp. Substrates with Agar, Agar + Glucose, Agar + Salts, Agar + Yeast extracts and Agar + Glucose + Salts + Yeast extracts were prepared. Ammonium acetate buffer was added on top of the substrates and incubated for $10 \mathrm{~min}$. the buffer was removed and filtrated. SF spectra of the filtrates $\left(\Delta \lambda=60 \mathrm{~nm}, \lambda_{\mathrm{Em}}=310-370 \mathrm{~nm}\right)$ were measured. (Figure. 3$)$. The substrates of Agar and Agar + Glucose did not have a significant fluorescence contribution. A small fluorescence contribution of 0.1 CPS was obtained from the Agar + Salts substrate, however, the shape of the peak was not similar to the peak of Trp. Interestingly, when all ingredients were added to the substrate, no significant fluorescence was observed. The results show that the presence of sugars and 
salts in the substrate prevents its extraction to the ammonium acetate buffer. We postulate that the reason for this could result from interaction between the proteins and the salts or sugars. Another reason may be the increase in the viscosity of the gel which limits the diffusion of the proteins to the upper surface of the substrate.

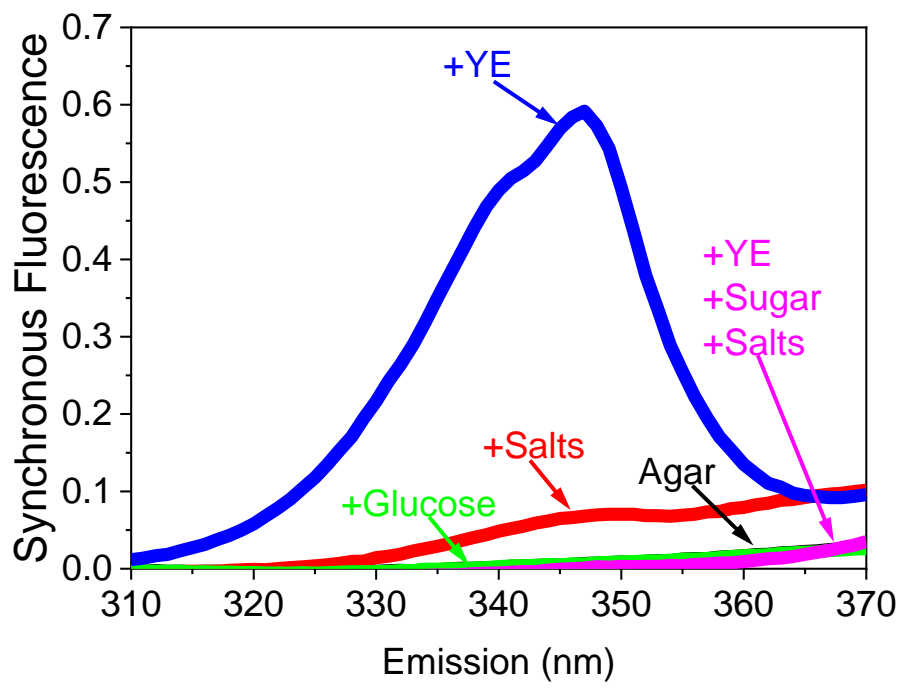

Fig. 3 - SF measurements of the ingredients of the new medium. SF of the ingridients of the new medium were measured to evaluate their fluorescence overlap with Trp. Agar (black), Agar + salts (red), Agar + glucose (green), Agar + yeast extract (blue).

\section{Identification of $\varphi x-174$ based on its Trp fluorescence fingerprint}

Using the LTS has eliminated the extraction of proteins from the substrate to the ammonium acetate buffer. However, proteins other fluorescent materials may be secreted from the E.coli cells or be released by dead or lysed cells. we wished to assess whether such materials are extracted into the Ammonium acetate buffer and whether their fluorescence overlaps with the spectral fingerprint of TRP. E.coli layer was grown on the LTS with or without addition of $\varphi x-174$. Amonium acetate buffer was added on the top of the dish for $10 \mathrm{~min}$. The buffer was removed and filtrated through $0.2 \mu \mathrm{m}$ filter. SF of the filtrates was measured. (Figure. 4). a fluorescence contribution between $330-370 \mathrm{~nm}$ was obtained from the sample without $\varphi x-174$. However, it's spectral shape was linear and clearly not originating from TRP. The samples with $\varphi x-174$ showed the spectral fingerprint of TRP. In order to evaluate the TRP concentration. The spectra of the sample without $\varphi x-174$ was substructed from the sample with it. a calibration curve of concentration vs. fluorescence intensity at $350 \mathrm{~nm}$ was prepared by measuring SF spectra of increasing Trp concentrations $(0.3,0.6,1.2$, and $1.8 \mu \mathrm{M})$. The Trp concentration of $\varphi \mathrm{x}-174$ was determined to be $0.44-/+0.03 \mu \mathrm{M}$. 
The results shows that when using the LTS substrate (instead of the conventional NA), it is possible to use SF to identify the TRP fluorescence contribution which originates only from $\varphi x-174$ and not from its environment.

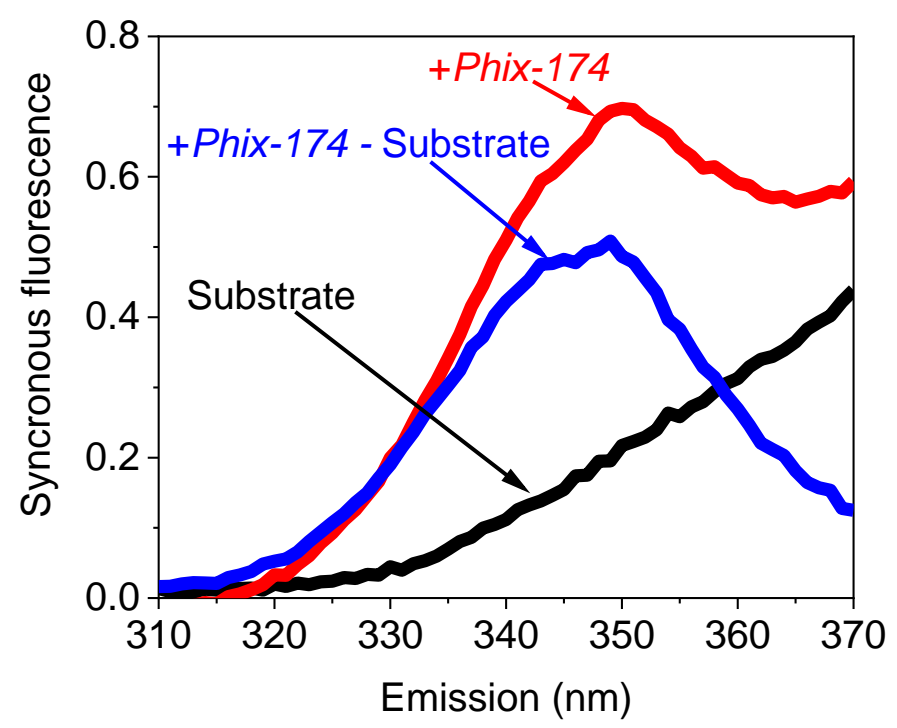

Fig. 4 - Identification of $\varphi x-174$ based on its Trp fluorescence fingerprint.

\section{Conclusions}

In this work, we present a new substrate for bacterial cultivation which consist significantly lower Trp content than NA which is frequently used. We show that by using this substrate as a platform for cultivation of E.coli and replication of the virus $\varphi x-174$, we enable the possibility to identify $\varphi x-174$ based on its intrinsic fluorescence which originates from its Trp content. This separation and identification method is simple and rapid and may be used in the future for identification of various viral species.

\section{Experimental}

\section{Replication of the virus $\varphi x-174$}

To replicate the virus $\varphi x-174$, we used the "double-layer" method. Briefly, in this method, a host bacterium (in high concentration) is grown into a solid medium together with infectious phage particles. After the phage is replicate itself in the bacterium, and lyse the bacterium cells, a visible clear zone (plaque) is formed. In our case, to E. coli CN13 (ATCC 700609, a nalidixic acid resistant strain) a $\varphi x$ 174 ( DSM 4497, Germany) was added and were grown on Nutrient Agar (NA) composed from Nutrient Broth (Difco, USA) with $0.7 \%$ Agar (Difco, USA). After over-night incubation $\left(37^{\circ} \mathrm{C}\right.$ ) a viral plaques were formed.

Purification of the virus $\varphi x-174$ from whole E.coli cells 
For separation of viral particles from whole E. coli cells, a $5 \mathrm{ml}$ of Ammonium acetate buffer (Ammonium acetate, Riedel-de Haen, Germany, $\mathrm{pH}=7,0.1 \mathrm{M}$ ) were added on the "double-layer" Agar (Petri dish of $100 \mathrm{~mm}$ diameter). The buffer was float on the Agar for $10 \mathrm{~min}$ and then it pumped out by syringe and filtered by PVDF sterile syringe filter ( $0.22 \mu$, Millipore, Ireland). As a control, the same procedure was done for "double-layer" Agar without addition of $\varphi x-174$ particles prior the incubation (named TEC).

\section{Preparation of the low trp conent medium}

The new media consisted from Glucose, $10 \mathrm{gr} / \mathrm{L}$ (Merck, Germany); $\mathrm{KH}_{2} \mathrm{PO}_{4}, 5 \mathrm{gr} / \mathrm{L}$ (Carlo Erba, Italy); $\mathrm{K}_{2} \mathrm{HPO}_{4} \cdot 3 \mathrm{H}_{2} \mathrm{O}, 5 \mathrm{gr} / \mathrm{L}$ (Merck, Germany); $\left(\mathrm{NH}_{4}\right)_{2} \mathrm{HPO}_{4}, 3 \mathrm{gr} / \mathrm{L}$ (Merck, Germany); $\mathrm{Na}_{2} \mathrm{HPO}_{4}$, $4.6 \mathrm{gr} / \mathrm{L}$ (Fluka, Switzerland); $\mathrm{MgSO}_{4} \cdot 7 \mathrm{H}_{2} \mathrm{O}, 0.6 \mathrm{gr} / \mathrm{L}$, (Merck, Germany); Yeast extract, $0.5 \mathrm{gr} / \mathrm{L}$ (Difco, USA); Agar, 7 gr/L (Difco, USA).

\section{References}

1. Salemi S, Markovic M, Martini G, D’Amelio R (2015) The expanding role of therapeutic antibodies. Int Rev Immunol 34:202-264 . https://doi.org/10.3109/08830185.2013.863304

2. Li C, Mori L, Valente ST (2021) The Block-and-Lock Strategy for Human Immunodeficiency Virus Cure: Lessons Learned from Didehydro-Cortistatin A. J Infect Dis 223:S46-S53 . https://doi.org/10.1093/infdis/jiaa681

3. Komiyama M (2021) Molecular-Level Anatomy of SARS-CoV-2 for the Battle against the COVID-19 Pandemic. Bull Chem Soc Jpn 94:1478-1490 . https://doi.org/10.1246/bcsj.20210030

4. Arias-Arias JL, MacPherson DJ, Hill ME, Hardy JA, Mora-Rodríguez R (2020) A fluorescence-activatable reporter of flavivirus NS2B-NS3 protease activity enables live imaging of infection in single cells and viral plaques. J Biol Chem 295:2212-2226 . https://doi.org/10.1074/jbc.RA119.011319

5. Crosson SM, Dib P, Smith JK, Zolotukhin S (2018) Helper-free production of laboratory grade AAV and purification by Iodixanol density gradient centrifugation. Mol Ther - Methods Clin Dev 10:1-7 . https://doi.org/10.1016/j.omtm.2018.05.001

6. Hadiji-Abbes N, Martin M, Benzina W, Karray-Hakim H, Gergely C, Gargouri A, Mokdad-Gargouri R (2013) Extraction and purification of hepatitis B virus-like M particles from a recombinant Saccharomyces cerevisiae strain using alumina powder. 
J Virol Methods 187:132-137 . https://doi.org/10.1016/j.jviromet.2012.09.023

7. Rocha JM (2021) Aqueous two-phase systems and monolithic chromatography as alternative technological platforms for virus and virus-like particle purification. $\mathrm{J}$ Chem Technol \\& Biotechnol 96:309-317 . https://doi.org/https://doi.org/10.1002/jctb.6595

8. Steger G, Riesner D (2018) Viroid research and its significance for RNA technology and basic biochemistry. Nucleic Acids Res 46:10563-10576 . https://doi.org/10.1093/nar/gky903

9. Popov VL, Tesh RB, Weaver SC, Vasilakis N (2019) Electron microscopy in discovery of novel and emerging viruses from the collection of the world reference center for emerging viruses and arboviruses (WRCEVA). Viruses 11: . https://doi.org/10.3390/v11050477

10. Mukherjee S, Boutant E, Réal E, Mély Y, Anton H (2021) Imaging viral infection by fluorescence microscopy: focus on HIV-1 early stage. Viruses 13: . https://doi.org/10.3390/v13020213

11. Carter and Saunders (2012) Virology: Principles and Applications (2nd Ed.)

12. Gensberger ET, Kostić T (2013) Novel tools for environmental virology. Curr. Opin. Virol. 3:61-68

13. Cheng MP, Papenburg J, Desjardins M, Kanjilal S, Quach C, Libman M, Dittrich S, Yansouni CP (2020) Diagnostic Testing for Severe Acute Respiratory SyndromeRelated Coronavirus 2: A Narrative Review. Ann. Intern. Med. 172:726-734

14. Ménard-Moyon C, Bianco A, Kalantar-Zadeh K (2020) Two-Dimensional MaterialBased Biosensors for Virus Detection. ACS Sensors 5:3739-3769 . https://doi.org/10.1021/acssensors.0c01961

15. Banerjee S, Maurya S, Roy R (2018) Single-molecule fluorescence imaging: Generating insights into molecular interactions in virology. J Biosci 43:519-540 . https://doi.org/10.1007/s12038-018-9769-y

16. Chojnacki J, Eggeling C (2018) Super-resolution fluorescence microscopy studies of human immunodeficiency virus. Retrovirology 15:41 
17. De Almeida Pondé RA (2013) Enzyme-linked immunosorbent/chemiluminescence assays, recombinant immunoblot assays and nucleic acid tests in the diagnosis of $\mathrm{HCV}$ infection. Eur. J. Clin. Microbiol. Infect. Dis. 32:985-988

18. Iha K, Inada M, Kawada N, Nakaishi K, Watabe S, Tan YH, Shen C, Ke L-Y, Yoshimura T, Ito E (2019) Ultrasensitive ELISA Developed for Diagnosis. Diagnostics 9:78 . https://doi.org/10.3390/diagnostics9030078

19. Cantalupo PG, Pipas JM (2019) Detecting viral sequences in NGS data. Curr Opin Virol 39:41-48 . https://doi.org/https://doi.org/10.1016/j.coviro.2019.07.010

20. Tisthammer KH, Dong W, Joy JB, Pennings PS (2021) Comparative analysis of within-host mutation patterns and diversity of Hepatitis Cvirus Subtypes 1a, 1b, and 3a. Viruses 13: . https://doi.org/10.3390/v13030511

21. Srivastava S, Upadhyay DJ, Srivastava A (2020) Next-Generation Molecular Diagnostics Development by CRISPR/Cas Tool: Rapid Detection and Surveillance of Viral Disease Outbreaks. Front Mol Biosci 7:582499 . https://doi.org/10.3389/fmolb.2020.582499

22. Singh S, Kumar V, Kapoor D, Dhanjal DS, Bhatia D, Jan S, Singh N, Romero R, Ramamurthy PC, Singh J (2021) Detection and disinfection of COVID-19 virus in wastewater. Environ. Chem. Lett. 1:3

23. Qian J, Boswell SA, Chidley C, Lu Z xiang, Pettit ME, Gaudio BL, Fajnzylber JM, Ingram RT, Ward RH, Li JZ, Springer M (2020) An enhanced isothermal amplification assay for viral detection. Nat Commun 11:1-10 . https://doi.org/10.1038/s41467-020-19258-y

24. Naveen KP, Bhat AI (2020) Development of reverse transcription loop-mediated isothermal amplification (RT-LAMP) and reverse transcription recombinase polymerase amplification (RT-RPA) assays for the detection of two novel viruses infecting ginger. J Virol Methods 282:113884 . https://doi.org/10.1016/j.jviromet.2020.113884

25. Obande GA, Singh KKB (2020) Current and future perspectives on isothermal nucleic acid amplification technologies for diagnosing infections. Infect. Drug Resist. 13:455483 
26. Shahzad A, Edetsberger M, Koehler G (2010) Fluorescence Spectroscopy: An Emerging Excellent Diagnostic Tool in Medical Sciences. Appl Spectrosc Rev 45:111. https://doi.org/10.1080/05704920903435375

27. Ruokola P, Dadu E, Kazmertsuk A, Hakkanen H, Marjomaki V, Ihalainen JA (2014) Raman Spectroscopic Signatures of Echovirus 1 Uncoating. J Virol 88:8504-8513 . https://doi.org/10.1128/jvi.03398-13

28. Lambert PJ, Whitman AG, Dyson OF, Akula SM (2006) Raman spectroscopy: The gateway into tomorrow's virology. Virol. J. 3:51

29. Santos MCD, Morais CLM, Nascimento YM, Araujo JMG, Lima KMG (2017) Spectroscopy with computational analysis in virological studies: A decade (20062016). TrAC - Trends Anal. Chem. 97:244-256

30. Shen F, Triezenberg SJ, Hensley P, Porter D, Knutson JR (1996) Critical amino acids in the transcriptional activation domain of the Herpesvirus protein VP16 Are solventexposed in highly mobile protein segments: an intrinsic florescence study. J Biol Chem 271:4819-4826 . https://doi.org/10.1074/jbc.271.9.4819

31. Lakowicz JR (2006) Fluorophores. In: Principles of Fluorescence Spectroscopy. Springer US, pp 63-95

32. Marinowic DR, Zanirati G, Rodrigues FVF, Grahl MVC, Alcará AM, Machado DC, Da Costa JC (2021) A new SYBR Green real-time PCR to detect SARS-CoV-2. Sci Rep 11:2224 . https://doi.org/10.1038/s41598-021-81245-0

33. Andrade-Eiroa Á, de-Armas G, Estela JM, Cerdà V (2010) Critical approach to synchronous spectrofluorimetry. I. TrAC - Trends Anal. Chem. 29:885-901

34. Liu Q, Grant G, Vo-Dinh T (2010) Investigation of synchronous fluorescence method in multicomponent analysis in tissue. IEEE J Sel Top Quantum Electron 16:927-940 . https://doi.org/10.1109/JSTQE.2009.2031162

35. Rubio S, Gomez-Hens A, Valcarcel M (1986) Analytical applications of synchronous fluorescence spectroscopy. Talanta 33:633-640 . https://doi.org/https://doi.org/10.1016/0039-9140(86)80149-7

36. Lloyd JBF, Evett IW (1977) Prediction of peak wavelengths and intensities in synchronously excited fluorescence emission spectra. Anal Chem 49:1710-1715 . 
https://doi.org/10.1021/ac50020a020

37. Patra D, Mishra AK (2002) Recent developments in multi-component synchronous fluorescence scan analysis. TrAC Trends Anal Chem 21:787-798 . https://doi.org/https://doi.org/10.1016/S0165-9936(02)01201-3

38. Lloyd JBF (1971) Synchronized Excitation of Fluorescence Emission Spectra. Nat Phys Sci 231:64-65 . https://doi.org/10.1038/physci231064a0

39. Ammor MS (2007) Recent advances in the use of intrinsic fluorescence for bacterial identification and characterization. J Fluoresc 17:455-459 . https://doi.org/10.1007/s10895-007-0180-6

40. Perinchery SM, Kuzhiumparambil U, Vemulpad S, Goldys EM (2010) The potential of autofluorescence spectroscopy to detect human urinary tract infection. Talanta 82:912917 . https://doi.org/https://doi.org/10.1016/j.talanta.2010.05.049

41. Sahar A, Boubellouta T, Dufour É (2011) Synchronous front-face fluorescence spectroscopy as a promising tool for the rapid determination of spoilage bacteria on chicken breast fillet. Food Res Int 44:471-480 . https://doi.org/https://doi.org/10.1016/j.foodres.2010.09.006

42. Sohn M, Himmelsbach DS, Barton FE, Fedorka-Cray PJ (2009) Fluorescence spectroscopy for rapid detection and classification of bacterial pathogens. Appl Spectrosc 63:1251-1255 . https://doi.org/10.1366/000370209789806993

43. Li R, Goswami U, King M, Chen J, Cesario TC, Rentzepis PM (2018) In situ detection of live-to-dead bacteria ratio after inactivation by means of synchronous fluorescence and PCA. Proc Natl Acad Sci U S A 115:668-673 . https://doi.org/10.1073/pnas.1716514115

44. Chen-Xi S, V. VGGR, Yew-Hoong GK (2017) Interaction of microcystis and Phix174 in the aquatic environment. J Environ Eng 143:4017011 . https://doi.org/10.1061/(ASCE)EE.1943-7870.0001208

\section{Acknowledgements}

This study has been supported by an internal fund (Micro Grants for the Technion Recycling Initiative, Technion, Israel). Yaniv Shlosberg is supported by the Schulich Graduate fellowship. 


\section{Conflicts of interest}

There are no conflicts to declare.

\section{Author contributions}

YF ,YS conceived the idea. YF and YS designed the experiments. YF and YS performed the main experiments. YF, YS and RA wrote the paper. IS and RA supervised the entire research project and provide funding. 